\title{
Evaluación del Desempeño de Aisladores Eléctricos de Distribución Cerámicos y Poliméricos bajo Biocontaminación por Hongos
}

\author{
Herbert E. Rojas ${ }^{(1) \star}$ y Harvey D. Rojas ${ }^{(2,3)}$ \\ (1) Univ. Distrital Francisco José de Caldas, Grupo de Compatibilidad e Interferencia Electromagnética \\ (GCEM-UD), Cra 7 \# 40-53, piso 5, Bogotá D.C., Colombia, (e-mail: herojasc@udistrital.edu.co) \\ (2) Centro de Electricidad Electrónica y Telecomunicaciones, Servicio Nacional de Aprendizaje SENA, \\ Grupo de Investigación del CEET - SENA (GICS), Av. Cra 30 \# 17B-25-Sur, Bogotá D.C., Colombia. \\ (Email: davidrc@misena.edu.co). \\ (3) Ingeniería Electrónica, Universidad Manuela Beltrán. Avenida Circunvalar № 60-00, Bogotá D.C., \\ Colombia, (E-mail: harvey.rojas@docente.umb.edu.co)
}

* Autor a quien debe ser dirigida la correspondencia

Recibido Ago. 13, 2016; Aceptado Oct. 7, 2016; Versión final Nov. 11, 2016, Publicado Abr. 2017

\begin{abstract}
Resumen
Este artículo presenta un estudio sobre el desempeño eléctrico de aisladores cerámicos (ANSI 52-1, ANSI 55-2) y poliméricos (ANSI DS-15) usados en sistemas eléctricos de media tensión (entre $7 \mathrm{kV}$ y 35 $\mathrm{kV}$ ) ante el crecimiento de los hongos Phanerochaete chrysosporium y Penicillium Sp. en su superficie. La contaminación biológica de aisladores eléctricos es una problemática que ha sido reportada en áreas tropicales de Europa, Asia y Oceanía y puede reducir la confiabilidad de los sistemas eléctricos de potencia. La biocontaminación se realizó en Bogotá, Colombia durante 50 días consecutivos (para cada microorganismo) usando una cámara que controla la luz, la humedad y la temperatura. Durante la investigación se realizaron pruebas de tensión de flameo y corrientes de fuga. Después de la biocontaminación se reportaron reducciones en las tensiones de flameo de $21 \%$ y $8 \%$ para aisladores cerámicos y poliméricos, respectivamente. En todos los casos, las corrientes de fuga aumentaron por encima del $20 \%$.
\end{abstract}

Palabras clave: biocontaminación; aisladores de distribución; tensión de flameo; corriente de fuga; Penicillium Sp.; Phanerochaete chrysosporium

\section{Performance Evaluation of Ceramic and Polymeric Electrical Distribution Insulators under Fungus Biocontamination}

\begin{abstract}
This paper presents a study on the electrical performance of ceramic (ANSI 52-1, ANSI 55-2) and polymeric (ANSI DS-15) insulators used in medium voltage electrical systems (between $7 \mathrm{kV}$ y $35 \mathrm{kV}$ ) when fungi growth of Phanerochaete chrysosporium and Penicillium Sp. on their surface is presented. Biological contamination of electrical insulators is a problem that has been reported in tropical areas in Europe, Asia and Oceania and it may reduce the reliability of electric power systems. The biocontamination was conducted in Bogota, Colombia for 50 consecutive days (for each microorganism) using a chamber that controls light, moisture and temperature. Laboratory tests of flashover voltages and leakage currents were performed. After fungus biocontamination reductions in flashover voltages about $21 \%$ and $8 \%$ were reported for ceramic and polymeric insulators, respectively. In all cases, the leakage currents increased by $20 \%$ or higher.
\end{abstract}

Keywords: biocontamination; distribution insulators; flashover voltage; leakage current; fungus biocontamination 


\section{INTRODUCCIÓN}

Los aisladores eléctricos son elementos que proporcionan separación física (distancias de seguridad) y soporte mecánico a conductores energizados y son ampliamente usados en sistemas de distribución y transmisión de energía en todo el mundo. Estos elementos se encuentran a la intemperie y funcionan bajo diferentes condiciones ambientales que los hacen propensos a múltiples tipos de contaminación (ambiental, industrial y biológica) (Amin y Salman, 2006). En este contexto, diversos estudios han reportado que el crecimiento y desarrollo de microorganismos puede presentarse en aisladores eléctricos de diferentes materiales (vidrio, cerámica, polímero, resina epóxica, etc.) y ponen de manifiesto que la colonización microbiana sobre la superficie de estos elementos eléctricos puede producir cambios en su normal funcionamiento. Estas alteraciones en los aisladores incluyen la reducción de su hidrofobicidad, su resistencia superficial y sus tensiones de flameo (Bengtsson y Grönlund, 2005; Lazar et al., 2008; León et al., 2014; Rojas et al., 2015; Wallström et al., 2005).

De manera complementaria, algunas investigaciones muestran que el desarrollo microbiano sobre aisladores eléctricos se beneficia de las condiciones ambientales propias de zonas tropicales tales como: la intensa radiación solar, los largos periodos de lluvias, la alta humedad relativa y las elevadas temperaturas (Amin y Salman, 2006; Atarijabarzadeh et al., 2011; Fernando y Gubanski, 2010; Meijer et al., 2011; Rojas et al., 2015; Sylvestre et al., 2002). Sin embargo, debido que la contaminación microbiana es un proceso que no está limitado a una región específica del planeta, varía según el tipo de microrganismo, y puede desarrollarse bajo diferentes condiciones ambientales, el impacto que el crecimiento de microorganismos genera sobre el desempeño de los aisladores eléctricos aún no se conoce por completo.

El hongo blanco de la podredumbre Phanerochaete chrysosporium y el hongo filamentoso Penicillium Sp. son microorganismos de forma avanzada (en comparación con otros como las bacterias) que se encuentran suspendidos en el aire y están presentes en casi todas partes del planeta. Estos hongos, junto con otras comunidades microbianas pueden descomponer la materia orgánica disponible en su entorno y forman parte importante del ciclo de nutrientes en ecosistemas complejos como bosques o selvas (Effron et al., 2012). Además, bajo ciertas condiciones climáticas y la disposición de ciertos nutrientes en el ambiente, los hongos generan procesos metabólicos que pueden degradar materiales aislantes sintéticos como los polímeros y las siliconas (Bennet et al., 2002; liyoshi et al., 1998).

Ahora bien, aunque estos estudios son evidencia de la afectación que sufren algunos materiales aislantes debido al crecimiento de hongos en su superficie, a nivel regional (Centro y Suramérica) no existen pruebas del impacto que la colonización microbiana puede causar en los aisladores eléctricos. Este artículo presenta un estudio sobre el desempeño eléctrico de aisladores cerámicos de suspensión ANSI 52-1, aisladores cerámicos tipo pin ANSI 55-2 y aisladores poliméricos ANSI DS-15, usados en sistemas eléctricos de media tensión (entre $7 \mathrm{kV}$ y $35 \mathrm{kV}$ ), ante el crecimiento en su superficie de los hongos Phanerochaete chrysosporium y Penicillium Sp. La biocontaminación se realizó en Bogotá, Colombia durante 50 días consecutivos (para cada microorganismo) usando una cámara que monitorea y controla las condiciones ambientales necesarias para asegurar el crecimiento y desarrollo de los hongos.

A lo largo de este trabajo se realizaron pruebas de laboratorio para evaluar el rendimiento eléctrico de cada uno de los aisladores biocontaminados. Las pruebas realizadas incluyen la medición de la tensión de flameo en seco y en húmedo (a baja frecuencia) y las corrientes de fuga. Para el desarrollo de estas pruebas experimentales se siguieron los procedimientos descritos en la norma técnica colombiana NTC 1285 (ICONTEC, 2002) y el estándar americano IEEE-4 (IEEE Standards, 2013). Adicionalmente, se presenta una comparación de los resultados experimentales obtenidos antes y después de la contaminación con los hongos con el objetivo de establecer en qué proporción se ve afectado el rendimiento de los aisladores a causa del crecimiento microbiano sobre su superficie.

\section{CONTAMINACION BIOLÓGICA}

Para el desarrollo de este trabajo fueron utilizados como agentes contaminantes los hongos Phanerochaete chrysosporium y Penicillium Sp. Estos microorganismos fueron seleccionados teniendo en cuenta que son hongos comunes que se encuentran generalmente suspendidos en el aire, tienen la capacidad de crecer sobre una gran variedad de sustratos y establecen colonias bajo casi todas las condiciones ambientales, en especial, en las zonas tropicales (Mandigan et al., 2014; Vega y Franco, 2013).

\section{Phanerochaete chrysosporium}

El Phanerochaete chrysosporium es un microorganismo que pertenece al reino fungi y es conocido como uno de los hongos de la podredumbre blanca por el aspecto que genera al desarrollarse sobre materiales 
orgánicos e inorgánicos (Hammel y Kapich, 2002; Have y Teunissen, 2001). Este hongo tiene la capacidad de degradar gran variedad de compuestos y puede desarrollarse en ambientes con temperaturas hasta 40 ${ }^{\circ} \mathrm{C}$. Por esta razón, puede ser encontrado en selvas y bosques tropicales participando en la degradación de árboles y plantas (Mandigan et al., 2014). La Figura 1(a) muestra una cepa del hongo Phanerochaete chrysosporium, el cual fue cultivado en una caja de Petri antes de su implantación en los aisladores.

\section{Penicillium Sp.}

Los hongos del género Penicillium Sp. son uno los géneros biológicos más grande del planeta y pueden encontrarse en casi todas partes, en especial, en suelos húmedos y materiales orgánicos (Gorur y Montesinos, 2003; R. Gorur, 2015). Pueden crecer bajo condiciones de temperatura variable (entre $5^{\circ} \mathrm{C} \mathrm{y}$ $37^{\circ} \mathrm{C}$ ) y no presentan variaciones debido a las estaciones, aunque su condición óptima de desarrollo es cercana a los $23^{\circ} \mathrm{C}$ y con humedades superiores al $80 \%$. Los hongos Penicillium $\mathrm{Sp}$. se relacionan generalmente con propósitos medicinales y la comercialización de antibióticos, aunque algunas especies son responsables de la degradación de frutas, hortalizas, quesos, pan, cereales y otros alimentos (Kyanko et. al., 2010). Además, poseen algunas características particulares dentro de las que resalta el ser microorganismos versátiles y oportunistas. Una cepa de este hongo se muestra en la Figura 1(b).

Generalmente, los hongos Penicillium Sp. forman colonias de rápido crecimiento con la apariencia de terciopelos y forman estructuras tubulares llamadas hifas que se ramifican y cubren amplias zonas. Se conocen como hongos grises, verdes o azules debido al color de las manchas que forman durante su desarrollo. Estos hongos son capaces de degradar materiales como papel y algunos pegamentos y algunas especies pueden poner en riesgo la salud de los seres humanos afectando las vías respiratorias y la piel (Mandigan et al., 2014).

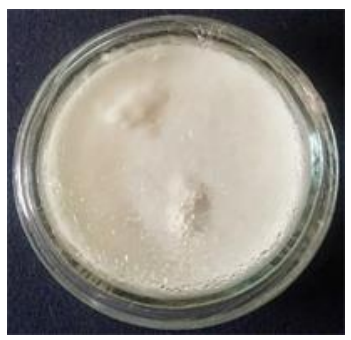

(a)

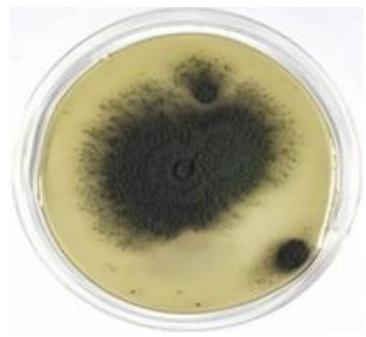

(b)

Fig. 1: Hongos seleccionados en caja de Petri (a) Phanerochaete chrysosporium; (b) Penicillium Sp.

\section{MATERIALES Y MÉTODOS}

Para este estudio, se seleccionaron tres tipos de aisladores de media tensión: cerámico de suspensión (ANSI 52-1), cerámico tipo pin (ANSI 55-2) y polimérico (ANSI DS-15). Los aisladores cerámicos están compuestos de porcelana eléctrica con una composición aproximada de $43 \%$ de arcilla $\left(\mathrm{Al}_{2} \mathrm{O}_{3} \cdot 2 \mathrm{SiO}_{2} \cdot 2 \mathrm{H}_{2} \mathrm{O}\right)$, $26 \%$ de óxido de silicio $\left(\mathrm{SiO}_{2}\right), 24 \%$ de feldespato $\left(\mathrm{K}_{2} \mathrm{O} \cdot \mathrm{Al}_{2} \mathrm{O}_{3} \cdot 6 \mathrm{SiO} 2\right)$ y $7 \%$ de Caolinita $\left(\mathrm{Al}_{2} \cdot \mathrm{Si} \cdot \mathrm{O}_{5} \cdot(\mathrm{OH})_{4}\right)$. Por otro lado, los aisladores poliméricos seleccionados están fabricados de goma de silicona de alto desempeño $\left(\mathrm{CH}_{3} \cdot \mathrm{SiO}\right)$. Estos aisladores fueron elegidos considerando que son frecuentemente usados en sistemas de distribución de media tensión (entre $7 \mathrm{kV}$ y $35 \mathrm{kV}$ ) en Colombia, Ecuador, Perú, Chile, Brasil, Estados Unidos y otros países de América, Asia y Europa. La Figura 2 muestra una imagen de los aisladores (cerámicos y poliméricos) usados en las pruebas, mientras sus características técnicas más importantes se resumen en la Tabla 1.

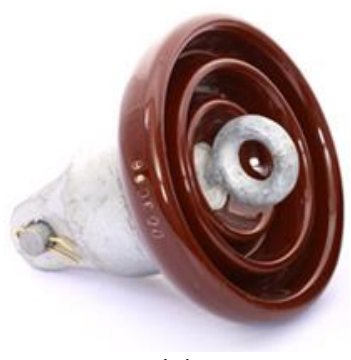

(a)

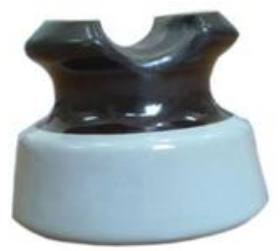

(b)

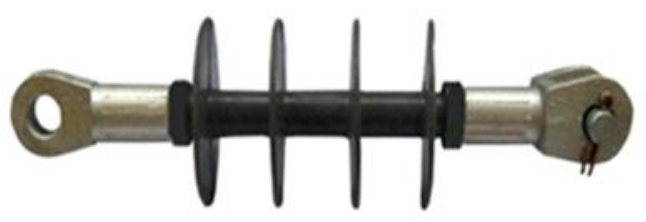

(c)

Fig. 2: Aisladores seleccionados (a) cerámico de suspensión ANSI 52-1;

(b) cerámico tipo pin ANSI 55-2; (c) polimérico ANSI DS-15 
Tabla 1: Características de los aisladores seleccionados (en condiciones estándar)

\begin{tabular}{|c|c|c|c|c|c|c|}
\hline Tipo de Aislador & Material & Discos & $\begin{array}{c}\text { Distancia de } \\
\text { fuga }[\mathrm{mm}]\end{array}$ & $\begin{array}{c}\text { Tensión } \\
\text { nominal }[\mathrm{kV}]\end{array}$ & $\begin{array}{c}\text { Tensión flameo } \\
\text { en seco }[\mathrm{kV}]\end{array}$ & $\begin{array}{c}\text { Tensión flameo en } \\
\text { húmedo }[\mathrm{kV}]\end{array}$ \\
\hline ANSI 52-1 & Cerámico & N.T. & 178 & 13.2 & 60 & 30 \\
\hline ANSI 55-2 & Cerámico & N.T. & 127 & 7.2 & 45 & 25 \\
\hline ANSI DS-15 & Polimérico & 4 & 401 & 15 & 90 & 65 \\
\hline
\end{tabular}

En la etapa inicial, los aisladores eléctricos usados durante el experimento fueron desinfectados y aislados del medio ambiente para evitar el contacto con agentes externos, y así, realizar los ensayos eléctricos de referencia. Una vez expuestos los aisladores al proceso de biocontaminación, y para evitar su alteración física por roturas, perdida de esmalte y/o cambios en la colonización microbiana, cada uno de los aisladores fue almacenado en un recipiente plástico y transportado de manera individual al laboratorio de alta tensión.

\section{Cámara de ambiente controlado y desarrollo biológico}

El crecimiento de los microorganismos sobre materiales aislantes expuestos a condiciones ambientales puede tomar periodos extensos que van desde algunos meses hasta varios años (Amin y Salman, 2006; Fernando y Gubanski, 2010). Para acelerar el proceso de colonización microbiana, y siguiendo la metodología propuesta por Dernfalk y Gubanski (2004), fue diseñada y construida una cámara para controlar la temperatura, la humedad relativa y la luz en su interior. Estas variables ambientales fueron identificadas como las más relevantes para el crecimiento y desarrollo de diversos microorganismos (hongos, algas y líquenes) después de comparar y analizar los resultados presentados por Sylvestre et al. (2002), Dernfalk y Gubanski (2004) y Fernando y Gubanski (2010). Los elementos que componen la cámara se muestran en la Figura 3(a).

La cámara fue fabricada en policarbonato transparente con dimensiones de $0.9 \mathrm{~m} \times 0.7 \mathrm{~m} \times 0.8 \mathrm{~m}$, lo cual brinda un espacio suficiente para instalar hasta nueve aisladores por cada ciclo de contaminación biológica. En su interior, cuenta con dos lámparas cerámicas Exo Terra® PT2044-40W para mantener la temperatura entre $23^{\circ} \mathrm{C}$ y $28^{\circ} \mathrm{C}$ y se instalaron dos boquillas de un sistema de rocío Exo Terra ${ }^{\circledR}$ Monsoon RS400 para garantizar una humedad relativa superior al $80 \%$. Adicionalmente, las condiciones de iluminación se ajustaron proporcionando periodos de 12 horas de luz y 12 horas de oscuridad mediante el uso de dos lámparas fluorescentes Repti Glo 2.0® PT2151. Estas lámparas tienen una potencia de $20 \mathrm{~W}$ y emiten luz en el espectro visible con longitudes de onda entre 380 [nm] y $640[\mathrm{~nm}]$.

Con el propósito de mantener las variables ambientales en los rangos definidos, el sistema fue automatizado usando una plataforma de hardware libre Arduino®. El monitoreo de la humedad relativa y la temperatura se realizó mediante dos sensores RHT-03. La información proveniente de los sensores se registra en el microcontrolador cada 30 segundos y es comparada con los valores de referencia. Una vez evaluados los datos, el microcontrolador envía una señal digital a un relé que activa o desactiva las bombillas cerámicas, el sistema de rocío de alta presión o el sistema de iluminación ultravioleta. De esta manera, se mantienen estables las condiciones ambientales necesarias para el crecimiento de los hongos Phanerochaete chrysosporium y Penicillium Sp. Un esquema general del sistema que controla la temperatura y la humedad en el interior de la cámara se muestra en la Figura 3(b)

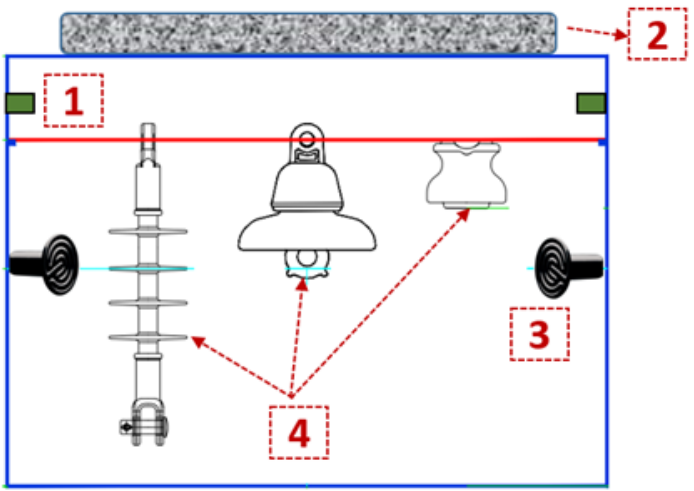

(a)

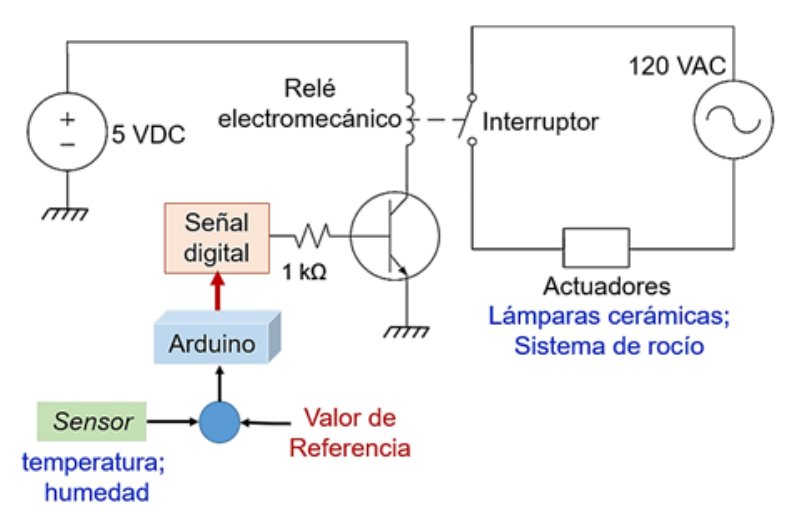

(b)

Fig. 3: Cámara de ambiente controlado (CAC). (a) Diagrama esquemático; (b) Esquema general de control (1) Boquillas de rocío; (2) Sistema de iluminación; (3) Lámparas de temperatura; (4) Aisladores 


\section{Contaminación de aisladores}

Para cada ciclo de contaminación (uno por cada hongo) se seleccionaron aleatoriamente tres unidades de aisladores eléctricos del tipo ANSI 52-1, ANSI 55-2 y ANSI DS-15. Los nueve aisladores fueron colocados al interior de la cámara, y posteriormente, se realizó la implantación del hongo en todos los elementos y sobre diversos puntos de su superficie. Esto se realizó con el fin de reducir la contaminación de los aisladores por diferentes agentes que pudiesen existir en el ambiente exterior.

Además de mantener estables las condiciones ambientales dentro de la cámara, se utilizó el medio de cultivo agar papa dextrosa (PDA, por sus siglas en inglés) para fomentar el proceso de colonización microbiana sobre la superficie de los aisladores. Asimismo, con el fin de proporcionar una adecuada exposición del microorganismo implantado a los nutrientes provistos y para garantizar una separación promedio entre elementos, los nueve aisladores fueron distribuidos de manera intercalada dentro de la cámara.

\section{RESULTADOS DE LAS PRUEBAS DE IDENTIFICACIÓN MICROBIANA}

En cada ciclo de contaminación, los aisladores permanecieron dentro la cámara durante un periodo de 50 días sin interrupción y bajo condiciones controladas de luz, temperatura y humedad relativa. Luego de este periodo, se evidenció sobre la superficie de todos los aisladores el crecimiento del microorganismo implantado. La Figura 4 y la Figura 5 evidencian la presencia de los hongos Phanerochaete chrysosporium y Penicillium Sp. respectivamente, sobre los aisladores de prueba. De los resultados obtenidos durante la contaminación, se resalta la acción que tiene la presencia del medio de cultivo PDA en el crecimiento de los hongos sobre los aisladores.

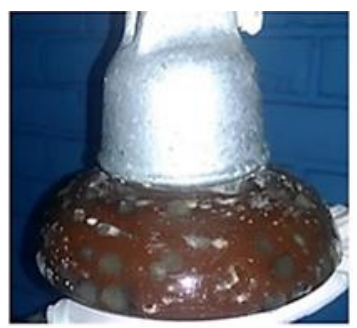

(a)

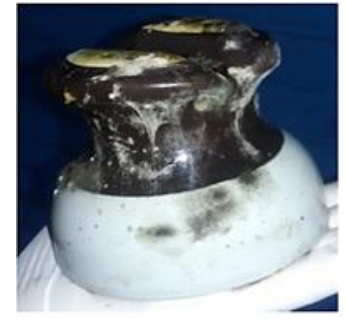

(b)

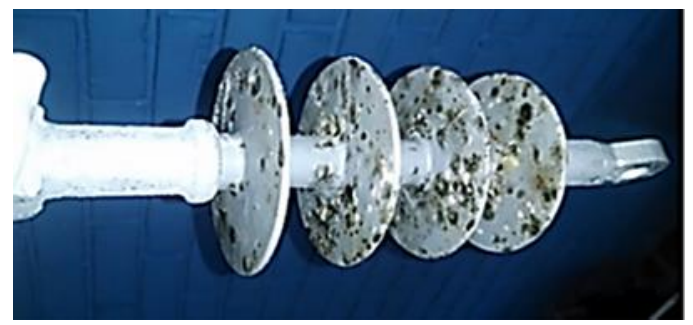

(c)

Fig. 4: Aisladores contaminados con Phanerochaete chrysosporium.

(a) ANSI 52-1; (b) ANSI 55-2; (c) ANSI DS-15

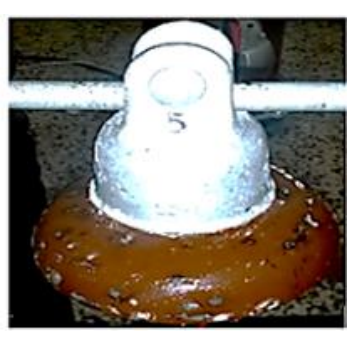

(a)

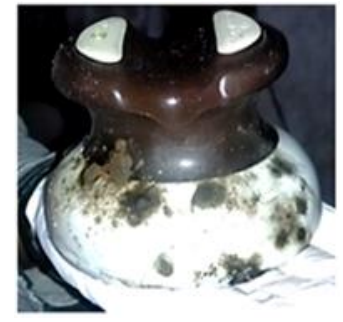

(b)

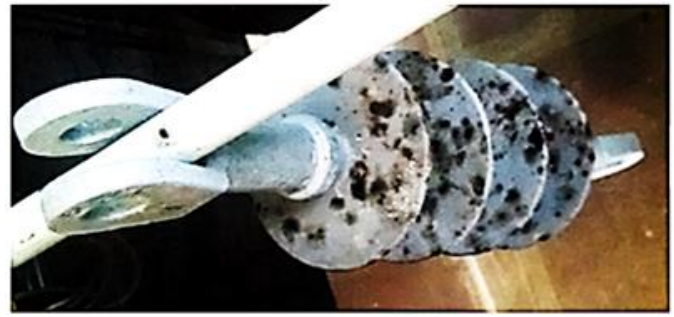

(c)

Fig. 5: Aisladores contaminados con Penicillium Sp. (a) ANSI 52-1; (b) ANSI 55-2; (c) ANSI DS-15

Luego de la contaminación biológica, todos los aisladores afectados por el hongo Phanerochaete chrysosporium presentaron manchas de color blanco, gris y verde oscura (ver Figura 4). En el caso de los aisladores de suspensión, el desarrollo del hongo es significativo sobre toda la superficie exhibiendo amplias zonas cubiertas por colonias microbianas, mientras que para los aisladores de pin ANSI 55-2 se observan brotes de crecimiento fúngico a menor escala concentrados en la zona del esmalte dieléctrico café. En el caso de los aisladores poliméricos, el crecimiento del microorganismo se evidencia fuertemente en ambas caras de los discos y en el núcleo que los une. Por otra parte, los aisladores expuestos al desarrollo de Penicillium Sp. presentaron formaciones circulares con tonalidad grisácea y verde oscura sobre su superficie (ver Figura 5). Adicionalmente, se observó un aumento en la captación de humedad, particularmente sobre las formaciones biológicas (manchas oscuras) que se unen con otras cercanas, estableciendo así, comunidades microbianas de mayor tamaño. 
En ambos ciclos de contaminación, las colonias de Phanerochaete chrysosporium y de Penicillium Sp. crecieron sobre la superficie de todos los aisladores sin excepción. Además, se observó que las colonias de ambos hongos se originan en puntos específicos y se expanden hacia el exterior del aislador, uniéndose con otras para formar colonias de mayor tamaño conocidas como biofilms (biopelículas), las cuales se adhieren a la superficie del elemento. No obstante, y pese a la notoria presencia de los hongos sobre la superficie (cerámica o polimérica) de los aisladores, en ninguno de los cultivos se observó desarrollo biológico sobre los herrajes, conectores o piezas metálicas.

Posteriormente, con la intervención y supervisión de dos laboratorios de microbiología certificados, en cada ciclo de biocontaminación se realizó una toma de muestras biológicas sobre los aisladores. Las muestras fueron tomadas usando hisopos estériles y trasportadas de manera individual a los laboratorios. Una vez allí, fueron sometidas a pruebas de identificación y conteo poblacional para avalar la presencia del microorganismo implantado en los aisladores. En la Tabla 2 y la Tabla 3 se muestran los resultados promedio de las pruebas de conteo biológico por cada tipo de aislador (para las tres muestras). Estos valores están expresados en cantidad de unidades formadoras de colonia (UFC) y aproximados al múltiplo de mil (1000) más cercano.

Tabla 2: Conteo poblacional primer ciclo (Phanerochaete chrysosporium)

\begin{tabular}{|c|c|c|c|c|}
\hline \multirow{2}{*}{ Microorganismo } & \multirow{2}{*}{ Identificación } & \multicolumn{3}{|c|}{ Conteo celular (UFC) } \\
\cline { 3 - 5 } & & ANSI 52-1 & ANSI 55-2 & ANSI DS-15 \\
\hline Phanerochaete chrysosporium & Positiva & 80000 & 12000 & 436000 \\
\hline Penicillium Sp. & Positiva & 4000 & 6000 & 14000 \\
\hline
\end{tabular}

Tabla 3: Conteo poblacional segundo ciclo (Penicillium Sp.)

\begin{tabular}{|c|c|c|c|c|}
\hline \multirow{2}{*}{ Microorganismo } & \multirow{2}{*}{ Identificación } & \multicolumn{3}{|c|}{ Conteo celular (UFC) } \\
\cline { 3 - 5 } & & ANSI 52-1 & ANSI 55-2 & ANSI DS-15 \\
\hline Penicillium Sp. & Positiva & 90000 & 200000 & 150000 \\
\hline
\end{tabular}

Aunque en ambos casos las pruebas de existencia de los hongos usados como agente contaminante fueron positivas, durante el primer ciclo de contaminación se observó también la presencia de Penicillium Sp. (ver Tabla 2). Esto se debe a que los hongos pertenecientes a este género permanecen suspendidos en partículas de polvo, y al ser microorganismos oportunistas, compiten por los mismos nutrientes que posee el medio de cultivo usado para promover el desarrollo de Phanerochaete chrysosporium. Pese a este resultado, la relación poblacional promedio entre Phanerochaete chrysosporium y Penicillium Sp para los tres aisladores fue de 23:1. Esta proporción asegura que los efectos que puedan producirse en el desempeño de los aisladores por la presencia de estos hongos se deberán a la presencia mayoritaria del hongo de la podredumbre blanca.

Al comparar los resultados mostrados en la Tabla 2 y la Tabla 3, se observa que para ambos hongos la mayor cantidad de UFC se presenta en los aisladores poliméricos. Este comportamiento se relaciona, por un lado, con el material del que se componen los aisladores ANSI DS-15, el cual puede ser un medio de crecimiento más atractivo para el desarrollo de los hongos; y por otro, con la mayor superficie que tiene este tipo de aisladores en comparación con los de tipo cerámico. Como resultado final, las pruebas biológicas muestran que aun aplicando procedimientos de seguridad e higiene siguiendo las normas establecidas, no es posible garantizar el crecimiento de un solo tipo de microorganismo sobre los aisladores. Sin embargo, aunque no fue requisito de este trabajo, en caso de exigirse esta condición, el aislamiento de la cámara sólo podrá conseguirse empleando cabinas de seguridad biológica o gabinetes de bioseguridad.

\section{RESULTADOS DEL DESEMPEÑO ELÉCTRICO Y DISCUSION}

Las pruebas de desempeño eléctrico se llevaron a cabo en el Laboratorio de Alta Tensión de la Universidad Distrital Francisco José de Caldas (LAT-UD) localizado en Bogotá, Colombia. Los ensayos realizados antes y después de la contaminación con los hongos incluyen la medición de tensión de flameo en seco (DFV), flameo en húmedo (WFV) y corrientes de fuga (LC).

\section{Pruebas eléctricas realizadas}

El procedimiento utilizado durante las pruebas es el descrito en la norma técnica colombiana NTC 1285 (ICONTEC, 2002) y el estándar IEEE-4 (IEEE Standards, 2013). El circuito usado para las pruebas eléctricas de referencia y los aisladores biocontaminados, se muestra en la Figura 6 . Algunas consideraciones adicionales, así como el diagrama eléctrico del montaje usado para las pruebas de tensión de flameo en húmedo, se muestran en (Rojas et al., 2015) 


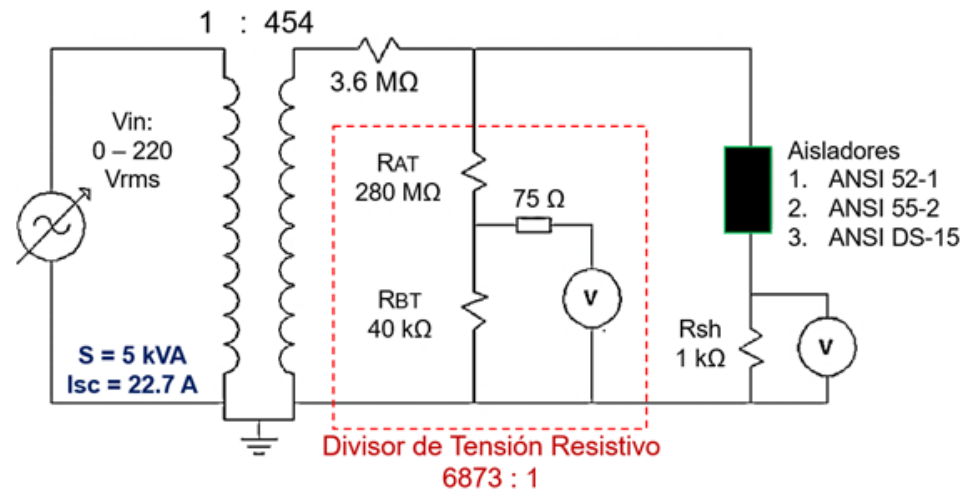

Fig. 6: Circuito usado para los ensayos DFV, WFV y LC. Adaptado de Rojas et al., 2015.

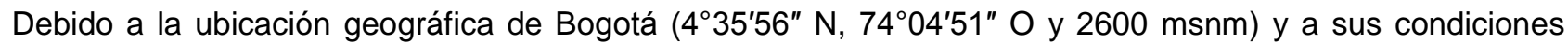
climáticas típicas (temperatura promedio: $14^{\circ} \mathrm{C}$, humedad relativa $75 \%$ ), se realizaron correcciones por condiciones atmosféricas para todas las pruebas realizadas. Esto con el propósito de obtener valores normalizados de acuerdo a los lineamientos de las normas técnicas empleadas. De esta manera, la tensión disruptiva esperada para cada aislador se estimó aplicando la siguiente relación:

$V d=\delta \times V \quad$ y además, $\quad \delta=\frac{P}{760} \times \frac{293}{273+T a}$

Donde, $\delta$ es la densidad relativa del aire para Bogotá, $P$ es la presión barométrica en $[\mathrm{mm}-\mathrm{Hg}]$, $T a$ es la temperatura en $\left[{ }^{\circ} \mathrm{C}\right]$ registrada, y $V$ es la tensión de flameo estipulada por el fabricante del aislador. De acuerdo a la norma, cada aislador fue sometido a cinco repeticiones (pruebas) y el valor de la tensión de flameo se calculó como la media aritmética de los valores registrados. Adicionalmente, para garantizar la fiabilidad de las pruebas, se realizaron las mediciones con una separación de entre 30 y 120 segundos y se verificó que la variación entre los valores medidos para cada aislador no excediera un valor absoluto de 5\%.

\section{Resultados de las pruebas de referencia}

Previo al desarrollo de las pruebas de referencia se marcaron los aisladores cerámicos de suspensión ANSI 52-1 (CS), cerámicos de pin ANSI 55-2 (CP) y los aisladores poliméricos ANSI DS-15 (PL) con una sigla y un número. Esto se hace para facilitar su identificación y posterior comparación. Los aisladores con índice 1, 2 y 3 fueron usados en el ciclo de contaminación con Phanerochaete chrysosporium, mientras los identificados con el número 4, 5 y 6 fueron contaminados con Penicillium Sp. Una vez rotulados los aisladores, se realizaron pruebas de tensión de flameo en seco y en húmedo (a baja frecuencia) y sus resultados se muestran en la Tabla 4 y la Tabla 5, respectivamente. En estas tablas, el valor de la tensión mostrada para cada muestra es el promedio de las cinco repeticiones a las que se sometió cada aislador, mientras la media y la desviación estándar calculadas se relacionan con el total de las pruebas realizadas (30 por cada tipo de aislador).

Tabla 4: Tensión de flameo en seco (DFV) antes la contaminación

\begin{tabular}{|c|c|c|c|c|c|c|c|c|}
\hline \multirow{2}{*}{$\begin{array}{c}\text { Tipo de } \\
\text { Aislador }\end{array}$} & \multicolumn{8}{|c|}{ Número de muestra (Tensión en $\mathrm{kV}$ ) } \\
\cline { 2 - 9 } & 1 & 2 & 3 & 4 & 5 & 6 & Media & $\sigma$ \\
\hline CS & 53.8 & 52.1 & 52.8 & 54.4 & 52.5 & 51.9 & 52.9 & 0.90 \\
\hline CP & 42.6 & 43.4 & 44.3 & 42.3 & 43.1 & 42.9 & 43.1 & 0.64 \\
\hline PL & 63.6 & 62.4 & 63.5 & 62.8 & 63.1 & 63.8 & 63.2 & 0.49 \\
\hline
\end{tabular}

Tabla 5: Tensión de flameo en húmedo (WFV) antes la contaminación

\begin{tabular}{|c|c|c|c|c|c|c|c|c|}
\hline \multirow{2}{*}{$\begin{array}{c}\text { Tipo de } \\
\text { Aislador }\end{array}$} & \multicolumn{8}{|c|}{ Número de muestra (Tensión en $\mathrm{kV}$ ) } \\
\cline { 2 - 9 } & 1 & 2 & 3 & 4 & 5 & 6 & Media & $\sigma$ \\
\hline CS & 34.2 & 32.0 & 32.3 & 32.5 & 31.7 & 32.0 & 32.5 & 0.82 \\
\hline CP & 21.8 & 21.4 & 21.5 & 21.8 & 21.7 & 21.9 & 21.7 & 0.18 \\
\hline PL & 60.0 & 57.9 & 58.7 & 57.8 & 58.6 & 57.7 & 58.5 & 0.79 \\
\hline
\end{tabular}

En términos generales, los resultados muestran que para aisladores cerámicos el valor promedio de la tensión de flameo en condiciones húmedas es 38\% menor con respecto a esta tensión en condiciones secas, mientras para los aisladores poliméricos la tensión de flameo en húmedo es $8 \%$ menor. Por otra parte, las mediciones de corriente de fuga se realizaron aplicando tensión desde $5 \mathrm{kV}$ y hasta el $75 \%$ de la tensión de flameo promedio para cada aislador (estimada previamente). Este límite de tensión se fijó para 
evitar flameos (disrupciones) sobre el aislador y aumentos excesivos en la corriente. Para esta prueba, se usó una resistencia shunt no-inductiva de $1 \mathrm{k} \Omega$ conectada en serie entre el aislador y el barraje de tierra (referencia del sistema), y se determinó la corriente midiendo su caída de tensión y usando la ley de Ohm. La Figura 7 muestra el comportamiento promedio de las corrientes de fuga para cada tipo de aislador.

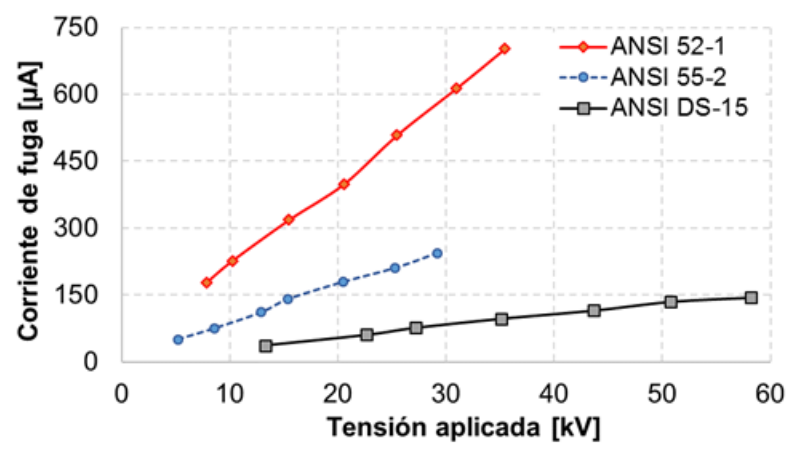

Fig. 7: Corrientes de fuga obtenidas durante las pruebas de referencia

Con respecto a las magnitudes de las corrientes de fuga para los tres tipos de aisladores, se obtuvo una diferencia entre muestras por debajo del 13\% para los aisladores cerámicos, mientras que en los aisladores poliméricos se presentó una diferencia entre muestras inferior al 7\%. En el caso de los aisladores ANSI 521 , se obtuvo en promedio una corriente de fuga máxima de $700 \mu \mathrm{A}$, mientras que para los aisladores tipo ANSI 55-2 las corrientes de fuga alcanzaron $245 \mu \mathrm{A}$ en promedio. Finalmente, para los aisladores poliméricos ANSI DS-15 las corrientes de fuga alcanzaron magnitudes cercanas a $150 \mu \mathrm{A}$. Estos resultados muestran que los aisladores cerámicos poseen una menor resistencia superficial que los aisladores poliméricos, lo cual se debe en parte a una menor de longitud de fuga en este tipo de aisladores.

\section{Resultados después de la contaminación biológica}

Finalizado el ciclo de biocontaminación, y una vez realizadas las pruebas de identificación y conteo poblacional, los aisladores contaminados fueron empacados por separado y transportados al laboratorio de alta tensión. Las pruebas realizadas en esta etapa se ejecutaron cumpliendo los mismos requerimientos técnicos y los procedimientos descritos para las pruebas de referencia. Los resultados de las pruebas de tensión de flameo en seco y húmedo de los aisladores eléctricos luego de la contaminación con el hongo Phanerochaete chrysosporium se muestran en la Figura 8 y Figura 9 respectivamente. En estas figuras se incluye el valor promedio calculado durante las pruebas de referencia (barra sólida izquierda) y se contrasta con los resultados promedio obtenidos para cada uno de los aisladores eléctricos contaminados después de realizadas las cinco pruebas individuales.

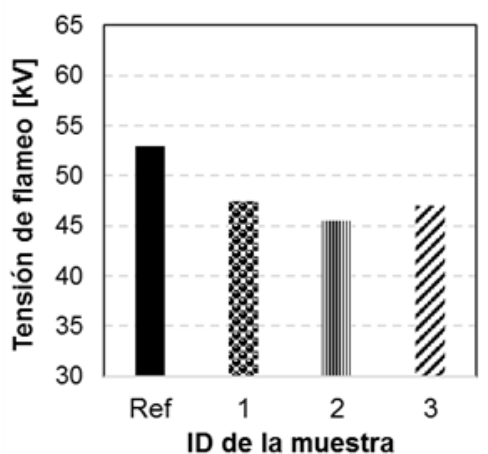

(a)

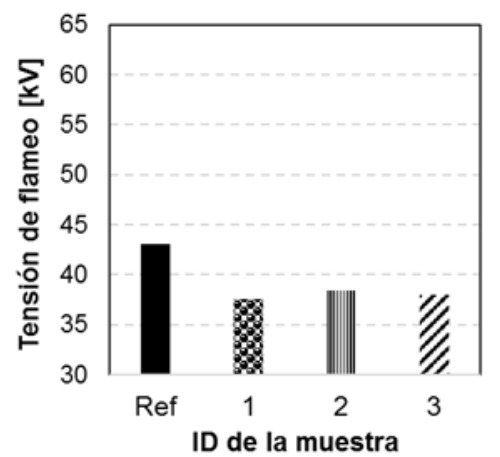

(b)

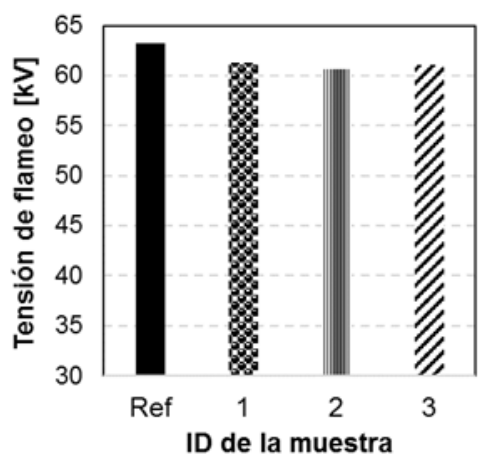

(c)

Fig. 8: Tensión de flameo en seco para aisladores contaminados con Phanerochaete chrysosporium (a) ANSI 52-1; (b) ANSI 55-2; (c) ANSI DS-15

Luego del primer ciclo de contaminación, se observa que el crecimiento de Phanerochaete chrysosporium sobre los aisladores disminuye la tensión de flameo de todas las muestras evaluadas. Al revisar los resultados de la Figura 8 se observa que las DFV de los aisladores ANSI 52-1 presentaron una reducción superior al 11\%, mientras que los aisladores ANSI 55-2 mostraron una reducción promedio de $12 \%$ y los aisladores poliméricos tuvieron una reducción de $4 \%$. Por otra parte, de los resultados obtenidos para la WFV (ver Figura 9) se resalta una reducción promedio de 10\% en todas las muestras, con una variación máxima de 14\% en los aisladores ANSI 55-2 y una reducción mínima de 8\% en los aisladores poliméricos. 


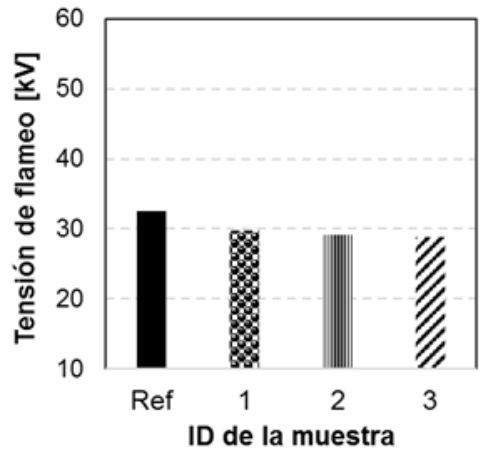

(a)

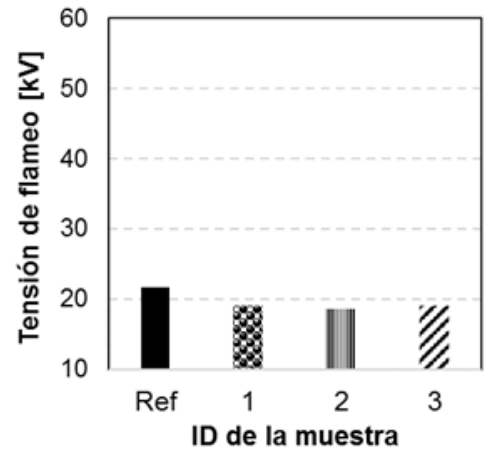

(b)

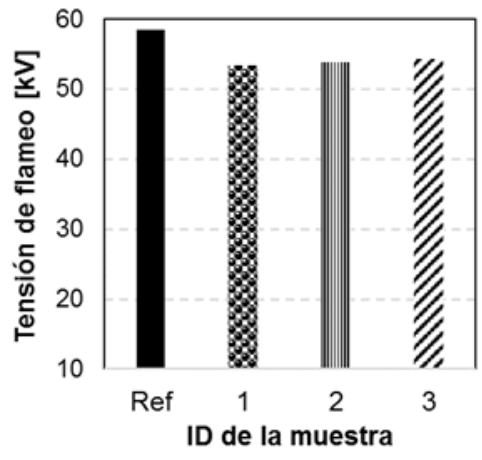

(c)

Fig. 9: Tensión de flameo en húmedo para aisladores contaminados con Phanerochaete chrysosporium (a) ANSI 52-1; (b) ANSI 55-2; (c) ANSI DS-15

Por otro lado, los valores promedio (para las 5 mediciones individuales) de las pruebas de tensión de flameo a frecuencia industrial para el segundo ciclo de contaminación se presentan en la Figura 10 y la Figura 11. Al igual que con el hongo de la podredumbre blanca, las pruebas luego de la contaminación con Penicillium $S p$. evidenciaron cambios en el desempeño de todos los aisladores. Para el caso de la DFV, se observaron reducciones promedio (respecto a su valor de referencia) de $21 \%, 13 \%$ y $6 \%$ para los aisladores ANSI $52-1$, ANSI 55-2 y ANSI DS-15 respectivamente. Por otro lado, las pruebas de WFV exhibieron una reducción promedio de $19 \%$ para los aisladores cerámicos y de 10\% para los aisladores poliméricos.

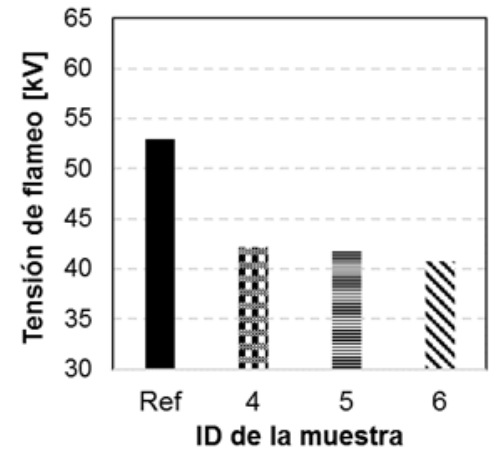

(a)

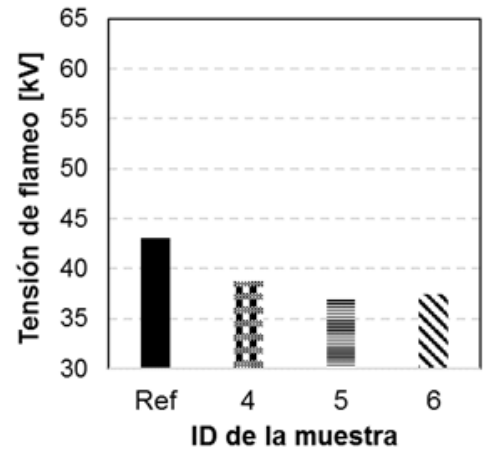

(b)

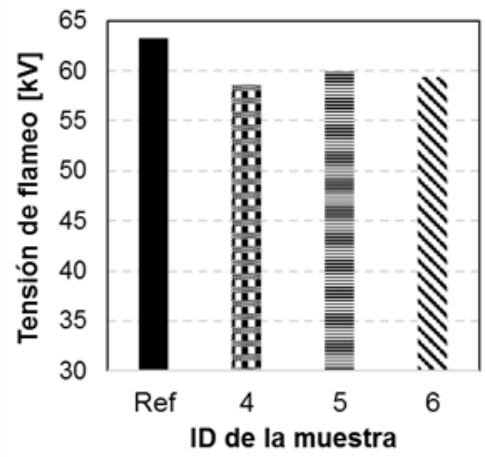

(c)

Fig. 10: Tensión de flameo en seco para aisladores contaminados con Penicillium Sp.

(a) ANSI 52-1; (b) ANSI 55-2; (c) ANSI DS-15

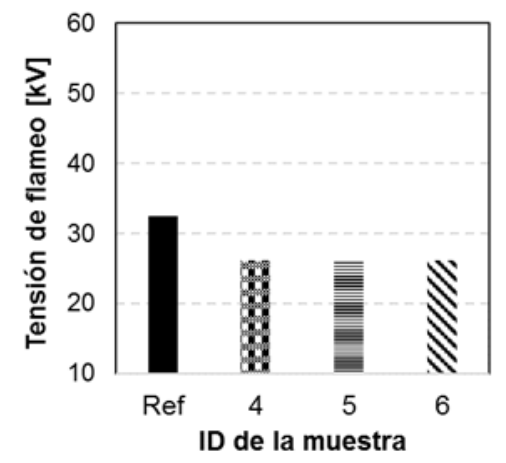

(a)

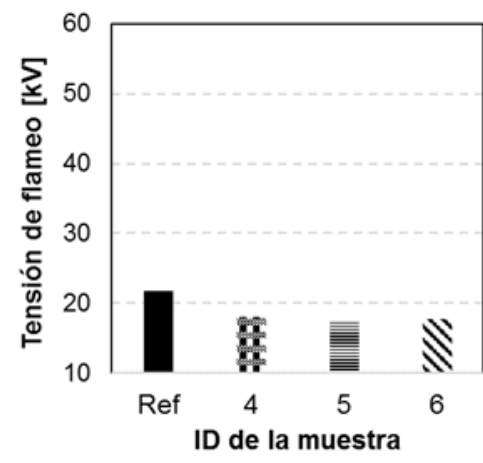

(b)

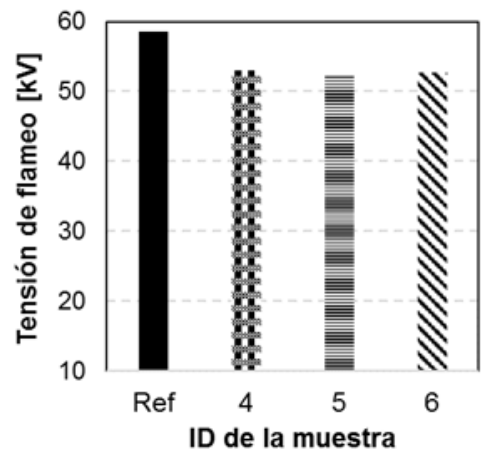

(c)

Fig. 11: Tensión de flameo en húmedo para aisladores contaminados con Penicillium Sp.

(a) ANSI 52-1; (b) ANSI 55-2; (c) ANSI DS-15

De manera similar a lo ocurrido con las tensiones de flameo, las corrientes de fuga medidas sobre los aisladores contaminados también mostraron cambios con respecto a sus valores de referencia. La Tabla 6 muestra el comportamiento promedio de las corrientes de fuga en todos los aisladores para cada ciclo de biocontaminación. A partir de los resultados obtenidos, se evidencia un crecimiento en la magnitud de las corrientes de fuga en todos los aisladores contaminados. Este comportamiento se relaciona con el aumento de la conductividad superficial de los aisladores debido a la formación de biopelículas microbianas sobre su superficie. 
Tabla 6: Corrientes de fuga para aisladores contaminados prueba de referencia (línea continua), prueba contaminación (línea punteada)

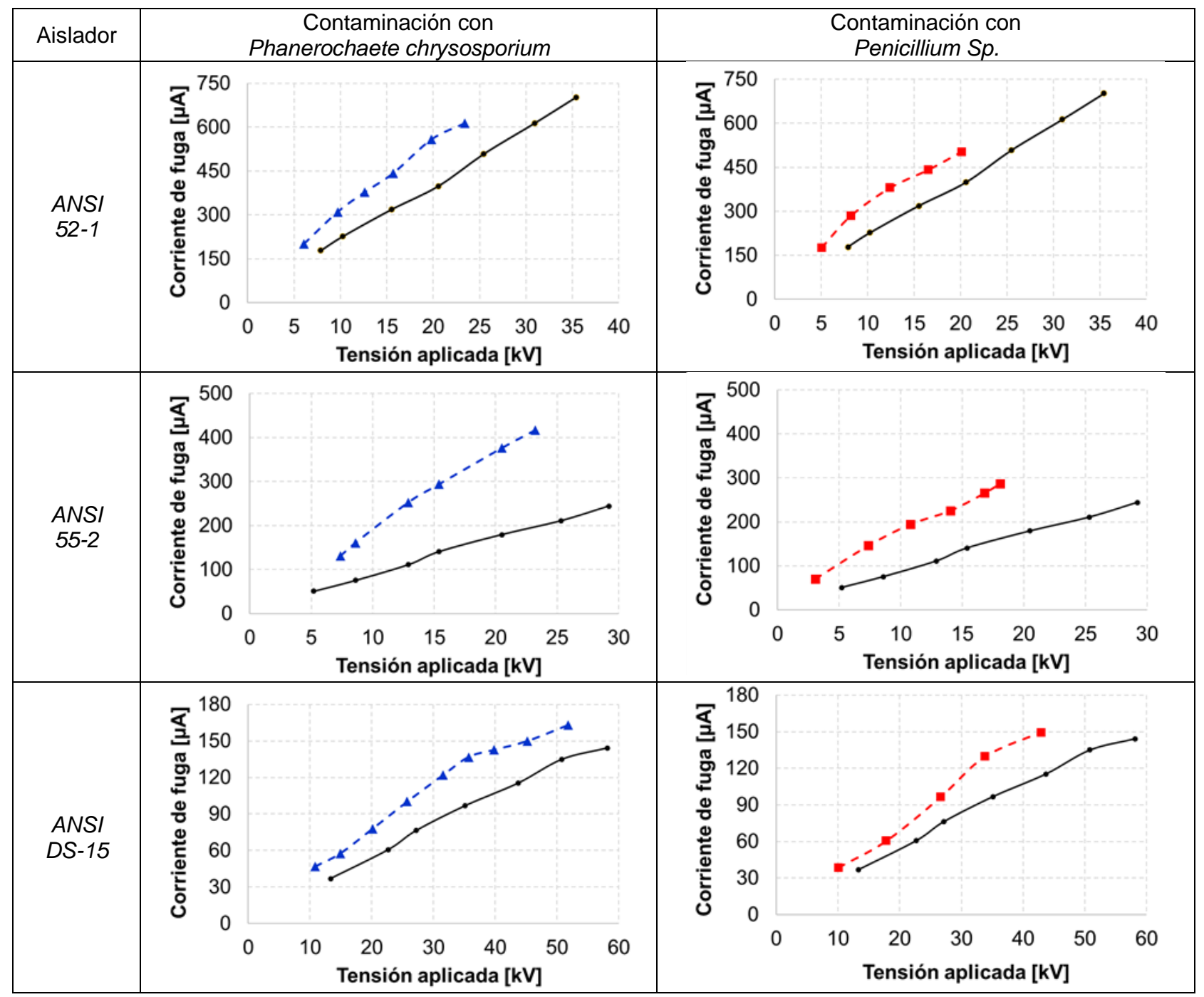

En el caso de la contaminación con Phanerochaete chrysosporium (columna izquierda, Tabla 6), las pruebas muestran que las corrientes de fuga alcanzan magnitudes cercanas a $610 \mu \mathrm{A}$ en los aisladores ANSI 52-1, mientras en los aisladores ANSI 55-2 las corrientes llegan hasta $420 \mu \mathrm{A}$ y en los aisladores poliméricos la corriente de fuga máxima es $160 \mu \mathrm{A}$. Por otra parte, para el caso del segundo ciclo de contaminación con Penicillium Sp. (columna derecha, Tabla 6), las corrientes de fuga en los aisladores ANSI 52-1 aumentaron en promedio $24 \%$ respecto a las pruebas de referencia, mientras en los aisladores ANSI $55-2$ el aumento llegó a un $48 \%$ y en los aisladores poliméricos ANSI DS-15 fue de 15\% aproximadamente.

Al analizar los resultados de la Tabla 6, se observa que la diferencia en el comportamiento de las corrientes de fuga se refleja en el valor de la tensión crítica que es aplicada antes de iniciarse el flameo sobre los aisladores. En términos generales,el valor máximo de la tensión aplicada sobre los aisladores contaminados se redujo un $35 \%$ para los aisladores cerámicos y un $25 \%$ para los aisladores poliméricos. En este contexto, se observa que en los aisladores poliméricos biocontaminados las corrientes de fuga alcanzan valores máximos similares a los medidos durante las pruebas de referencia. Sin embargo, las corrientes registradas durante la contaminación biológica fueron obtenidas aplicando menores niveles de tensión.

A partir de estos resultados, donde se evidencia el aumento de la corriente de fuga debido al crecimiento de hongos sobre los aisladores, siguiendo la metodología presentada por Rudolf (2009) es posible evaluar las pérdidas eléctricas que la biocontaminación puede generar en los sistemas eléctricos de potencia. Si bien es cierto que las corrientes de fuga máximas que se reportaron en este trabajo no exceden $0.6 \mathrm{~mA}$, en el caso de los aisladores poliméricos ANSI DS-15, que son usados de manera individual en sistemas de media tensión (hasta $35 \mathrm{kV}$ ), o en el caso de los aisladores de suspensión ANSI 52-1, que pueden ser usados en cadenas de aisladores para sistemas de transmisión (hasta $138 \mathrm{kV}$ ), el aumento en la corriente de fuga por la presencia de hongos puede generar pérdidas de $5 \mathrm{~W}$ y $58 \mathrm{~W}$ respectivamente. 
Con base en estas estimaciones, las pérdidas por biocontaminación en aisladores pueden ser comparables a otras pérdidas técnicas como las que se producen por efecto corona, aunque su valor puede ser despreciable en función de la potencia total transmitida. Sin embargo, se debe tener en cuenta que estas pérdidas pueden aumentar en función del número de aisladores contaminados que hagan parte del sistema de potencia. Por esta razón, estas pérdidas deberían ser incluidas en la estimación de las pérdidas técnicas totales, como se hace actualmente con aquellas pérdidas asociadas a la contaminación por polución o las relacionadas con las variaciones climáticas (sequia, lluvias ligeras, aguaceros o nieve).

Finalmente, aunque este trabajo presenta los resultados obtenidos para ensayos eléctricos efectuados a tres tipos de aisladores (ANSI 52-1, ANSI 55-2 y ANSI DS-15), es necesario replicar la metodología presentada en este artículo haciendo pruebas con otras referencias de aisladores, incluyendo otros microrganismos y analizando aisladores bajo condiciones de envejecimiento y desarrollo microbiano natural. Esto con el fin de realizar una evaluación generalizada del desempeño eléctrico de aisladores biocontaminados.

\section{CONCLUSIONES}

A lo largo de este artículo se presentó un estudio que evalúa el efecto provocado por el crecimiento de los hongos Phanerochaete chrysosporium y Penicillium $S p$. sobre el desempeño eléctrico de aisladores cerámicos y poliméricos usados en sistemas de distribución de media tensión. Las pruebas experimentales mostraron que el desarrollo fúngico sobre la superficie de todos los aisladores biocontaminados genera una reducción en las tensiones de flameo a frecuencia industrial (en seco y en húmedo) y un aumento en las corrientes de fuga. De manera específica, y en función del tipo material del aislador, las pruebas de laboratorio mostraron que los aisladores cerámicos (ANSI 52-1 y ANSI 55-2) presentan un mayor deterioro en su desempeño eléctrico en comparación con los aisladores poliméricos.

Luego del primer ciclo de contaminación se pudo evidenciar que la colonización microbiana sobre los aisladores fue realizada por los hongos Phanerochaete chrysosporium y Penicillium Sp. los cuales mostraron una relación poblacional de 23:1 a favor del hongo de la podredumbre blanca. En este caso, las pruebas mostraron una reducción promedio en las tensiones de flameo de $14 \%$ en los aisladores cerámicos, mientras que en los aisladores poliméricos la reducción máxima fue de $8 \%$. Por otra parte, para los aisladores contaminados exclusivamente con Penicillium Sp. (segundo ciclo de contaminación) se observó que la tensión de flameo se redujo entre $13 \%$ y $21 \%$ en los aisladores cerámicos y alcanzó solo un $10 \%$ en los aisladores poliméricos. Estos resultados evidencian que los aisladores contaminados con el hongo Penicillium Sp. presentaron mayores afectaciones en sus tensiones de flameo que aquellos contaminados con el hongo Phanerochaete chrysosporium.

Finalmente, al analizar la magnitud de las corrientes de fuga se observó en todos los casos un crecimiento en los valores registrados para los aisladores eléctricos contaminados. Al igual que con las tensiones de flameo, los mayores cambios en la corriente de fuga se evidencian en los aisladores cerámicos con un incremento entre $32 \%$ y $98 \%$ para los elementos contaminados durante el primer ciclo (Phanerochaete chrysosporium y de Penicillium Sp), y un aumento entre $24 \%$ y $48 \%$ para los aisladores con desarrollo único de Penicillium Sp. en su superficie. En términos generales, se observó que los aisladores contaminados durante el primer ciclo presentan mayor aumento en las corrientes fuga para los tres tipos de aisladores. Esto se debe en parte a la reducción la resistencia superficial de los aisladores debido a la formación de colonias de hongos a lo largo de toda la superficie de los aisladores.

\section{AGRADECIMIENTOS}

Este trabajo fue financiado por el CIDC de la U. Distrital Francisco a través del proyecto $N^{\circ} 2-5-355-13$ y de la Comisión de estudios posgraduales $\mathrm{N}^{\circ}$ 0002-2016. Adicionalmente, los autores extienden su agradecimiento al Centro de Electricidad, Electrónica y Telecomunicaciones (CEET) y al Sistema de investigación, desarrollo tecnológico e innovación del SENA (SENNOVA) quienes financiaron la publicación de este trabajo.

\section{REFERENCIAS}

Amin, M., y Salman, M., Aging of polymeric insulators (an overview), doi:10.1109/MEl.2007.386482, Reviews on Advanced Materials Science, 13(2), 93-116 (2006)

Atarijabarzadeh, S., Strömberg, E., y Karlsson, S., Inhibition of biofilm formation on silicone rubber samples using various antimicrobial agents, doi:10.1016/j.ibiod.2011.09.001, International Biodeterioration \& Biodegradation, 65(8), 1111-1118 (2011)

Bengtsson, M., y Grönlund, R., Fluorescence lidar imaging of fungal growth on high-voltage outdoor composite insulators, doi: 10.1016/j.optlaseng.2004.09.019, Optics and Lasers in Engineering 43, 43(6), 624-632 (2005) 
Bennet, J., Wunch, K., y Faison, B., Use of fungi biodegradation, in Manual of Environmental Microbiology, Second Edition, pp. 960-971, ASM Press Washington, D.C., USA (2002)

Dernfalk, A., \& Gubanski, S., Techniques for estimation of biological contamination on insulators using image analysis, doi: 10.1109/CEIDP.2004.1364336, Annual Report Conference on Electrical Insulation and Dielectric Phenomena, 659-662 (2004)

Effron, D. N., Sarti, G. C., Quinteros, M. C., \& Catán, S. I., Influencia de especies arbóreas implantadas sobre parámetros biológicos y bioquímicos en un suelo forestal de Chubut, Argentina, doi: 10.4067/S071807642012000200010, Información Tecnológica, 23(2), 87-92 (2012)

Fernando, M., y Gubanski, S., Ageing of silicone rubber insulators in coastal and inland tropical environment, doi: 10.1109/TDEI.2010.5448085, IEEE Trans. on Dielectrics and Electrical Insulation, 17(2), 326-333 (2010)

Gorur, R., One utility's experience with mold growth on silicone bushings. http://www.inmr.com/utilitysexperience-mold-growth-silicone-bushings/, Insul. News Mark. Report (2015)

Gorur, R., y Montesinos, J., Mold growth on nonceramic insulators and its impact on electrical performance, doi: 10.1109/TPWRD.2003.809681, IEEE Transactions on Power Delivery, 18(2), 559-563 (2003)

Hammel, K., y Kapich, A., Reactive oxygen species as agents of wood decay by fungi, doi: 10.1016/S01410229(02)00011-X, Enzyme and Microbial Technology, 445-453 (2002)

Have, R. T., y Teunissen, P., Oxidative mechanisms involved in lignin degradation by white-rot fungi, doi: 10.1021/cr000115I, Chemical Reviews-Columbus, 18 (2001)

IEEE Standards subcommitte, IEEE-4: Standard for High-Voltage Testing Techniques, doi:10.1109/IEEESTD.2013.6515981, IEEE Power Engineering Society 213, USA (2013)

liyoshi, Y., Tsutsumi, Y., y Nishida, T., Polyethylene degradation by lignin-degrading fungi and manganese peroxidase, doi: 10.1007/BF00521967, Journal of Wood Science, (44), 222-229 (1998)

Instituto Colombiano de Normas Técnicas (ICONTEC), NTC 1285 - Método de ensayo para aisladores de potencia eléctrica, https://tienda.icontec.org/wp-content/uploads/pdfs/NTC1285.pdf, Colombia (2002)

Kyanko, M. V., Russo, M. L., Fernández, M., y Pose, G., Efectividad del ácido peracético sobre la reducción de la carga de esporas de mohos causantes de pudrición poscosecha de frutas y hortalizas, doi: 10.1612/inf.tecnol.4299it.09, Información Tecnológica, 21(4), 125-130 (2010)

Lazar, E. E., Wills, R. B., Harris, A. M., y Spohr, L. J., Antifungal effect of gaseous nitric oxide on mycelium growth, sporulation and spore germination of the postharvest horticulture pathogens, Aspergillus niger, Monilinia fructicola and Penicillium italicum, doi:10.1111/j.1472-765X.2008.02373.x, Letters in Applied Microbiology, 46(6), 688-92 (2008)

León, A. F., Rojas, H. E., Cantor, L. F., y Pérez, C. D., Performance of Ceramic and Polymeric Distribution Insulators with Mold Growth on its Surface, doi: 10.1109/TDC-LA.2014.6955202, IEEE PES Transmission \& Distribution Conference and Exposition - Latin America, 1-6 (2014)

Mandigan, M., Martinko, Bender, K., y Buckley, D., Stahl, D., Brock Biology of Microorganisms, $14^{\text {th }}$ Edición, Pearson ED., London, England (2014).

Meijer, M., Houbraken, J. A., Dalhuijsen, S., Samson, R. A., y de Vries, R. P., Growth and hydrolase profiles can be used as characteristics to distinguish Aspergillus niger and other black aspergilli, doi:10.3114/sim.2011.69.02, Studies in Mycology, 69(1), 19-30 (2011)

Rojas, H. E., Pérez, C. D., León, A. F., y Cantor, L. F., Electrical performance of distribution insulators with chlorella vulgaris growth on its surface, doi: 10.15446/ing.investig.v35n1Sup.53578, Ingeniería e Investigación, 35 (Sup 1), 21-27 (2015)

Rudolf, L., Leakage currents and power losses on outdoor insulators under artificial rains, http://jtie.upol.cz/pdfs/jti/2009/02/19.pdf, Journal of Tech. and Information Education, 1(2), 100-108 (2009)

Sylvestre, A., Rain, P., y Rowe, S., Silicone rubber subjected to combined temperature and humidity effects, doi: 10.1109/CEIDP.2002.1048808, Annual Report Conference on Electrical Insulation and Dielectric Phenomena Silicone, 355-358 (2002)

Vega, A., y Franco, H., Productividad y calidad de los cuerpos fructíferos de los hongos comestibles Pleurotus pulmonarius RN2 y P. djamor RN81 y RN82 cultivados sobre sustratos lignocelulósicos, doi: 10.4067/S0718-07642013000100009, Información Tecnológica, 24(1), 69-78 (2013)

Wallström, S., Dernfalk, A., y Bengtsson, M., Image analysis and laser induced fluorescence combined to determine biological growth on silicone rubber insulators, doi:10.1016/j.polymdegradstab.2004.12.009, Polymer Degradation and Stability, 394-400 (2005) 